\title{
ESTUDO MULTIVARIADO DE SOLOS URBANOS DA CIDADE DE TERESINA
}

Márcio Cleto Soares de Moura, Ana Nídia Cunha Lopes, Graziella Ciaramella Moita e José Machado Moita Neto*

Departamento de Química, Universidade Federal do Piauí, Campus da Ininga, 64049-550 Teresina - PI, Brasil

Recebido em 4/1/05; aceito em 6/10/05; publicado na web em 14/3/06

\begin{abstract}
MULTIVARIATE STUDY OF URBAN SOILS OF TERESINA CITY. The concentrations of $\mathrm{Cu}, \mathrm{Pb}, \mathrm{Zn}, \mathrm{Cr}, \mathrm{Ni}, \mathrm{Al}, \mathrm{Mn}$ and $\mathrm{Fe}$ were measured by atomic absorption spectrometry, of 19 topsoil samples collected in the Teresina city urban area to discriminate natural and anthropic contributions and identify possible sources of pollution. The average concentrations of $\mathrm{Cu}, \mathrm{Zn}, \mathrm{Pb}$ and $\mathrm{Cr}$ of the urban soils were $6.11,8.56,32.12$ and $7,17 \mathrm{mg} / \mathrm{kg}^{-1}$, respectively. Statistical analysis techniques, such as principal component analysis (PCA) and hierarchical cluster analysis (HCA), were used to analyze the data. $\mathrm{Mn}, \mathrm{Ni}$ and $\mathrm{Cr}$ levels were interpreted as natural contributions, whereas $\mathrm{Pb}, \mathrm{Zn}$ and, in part, $\mathrm{Cu}$ were accounted for mainly by anthropic activities. High $\mathrm{Pb}$ levels were observed in the ancient avenues.
\end{abstract}

Keywords: soil; heavy metals; multivariate analysis.

\section{INTRODUÇÃO}

Os metais pesados estão naturalmente presentes nos solos em baixíssimas concentrações. Um aumento no teor destes metais apresenta efeitos significativos sobre a qualidade ambiental ${ }^{1}$. Muitos poluentes inorgânicos tóxicos ocorrem em solos e podem ser de origem natural, quando advêm do intemperismo da rocha de origem, ou devido à contribuição antrópica (ação direta ou indireta do homem). Alguns deles são tóxicos, mesmo em quantidades-traços, e sua toxicidade aumenta com o acúmulo no solo.

A poluição do solo por metais pesados está ligada a processos de acúmulo e transporte dessas espécies, que dependem de suas interações com o solo. A adsorção é um processo muito importante e responsável pelo acúmulo de metais pesados no solo² ${ }^{2}$ As interações metal-solo são bastante complexas, envolvendo reações de precipitação, dissolução, adsorção, dessorção, complexação e oxi-redução, nas fases orgânicas e inorgânicas dos solos ${ }^{3}$.

Essas reações são influenciadas pelos atributos dos solos, destacando-se $\mathrm{pH}$, potencial redox, textura, composição mineral, capacidade de troca de cátions (CTC), competição por sítios de adsorção, quelatação, teor e qualidade dos compostos orgânicos na fase sólida e na solução do solo, além das propriedades específicas de cada metal ${ }^{3,4}$.

Vários elementos químicos presentes nos solos são fisiologicamente essenciais para o crescimento e a reprodução vegetal. Os elementos que são necessários em grande quantidade são denominados macronutrientes. Os demais elementos essenciais que são exigidos em pequenas quantidades denominam-se micronutrientes ${ }^{5}$.

Os elementos chumbo, cádmio, ferro, cobre, manganês, zinco, crômio, cobalto e níquel são normalmente estudados em solos, pois seus níveis no ambiente podem ser traduzidos em índices confiáveis de fertilidade ou poluição ambiental. Metais como ferro, cobre, zinco e manganês são essenciais, isto é, são indispensáveis aos sistemas biológicos; cobalto e níquel são elementos que colaboram com o desenvolvimento das plantas, mas sua falta não é considerada um fator limitante, enquanto que, chumbo, crômio e cádmio são considerados não essenciais e até mesmo tóxicos em quantida-

*e-mail: jmoita@ufpi.br des-traços. Os metais essenciais podem também produzir efeitos tóxicos, quando são absorvidos em quantidades elevadas ${ }^{6,7}$.

$\mathrm{O}$ transporte de longo alcance de poluentes atmosféricos é considerado a principal fonte de metais pesados em áreas naturais ${ }^{8-10}$, pois as concentrações de alguns metais apresentam uma distribuição similar para regiões com diferentes densidades populacionais e atividades veiculares ${ }^{11,12}$. As quantidades de metais pesados no solo também estão relacionadas com fontes locais de poluição provenientes de atividades industriais, de restos de incineração e da combustão de combustíveis.

No estudo de metais pesados em solo destaca-se a técnica de espectrometria de absorção atômica. Com ela é possível quantificar uma grande variedade de elementos químicos em uma amostra.

A grande quantidade de informação química que pode ser obtida no solo remete à necessidade de se complementar a descrição estatística usando análise simultânea de todas as amostras e variáveis.

\section{Análise multivariada}

Os métodos estatísticos multivariados consideram as amostras e as variáveis em seu conjunto, permitindo extrair informações complementares que a análise univariada não consegue evidenciar. Um dos objetivos da utilização da análise multivariada é reduzir a representação dimensional dos dados, organizando-os em uma estrutura que facilita a visualização de todo o conjunto de dados.

As duas técnicas de análise multivariada mais conhecidas são a análise de agrupamento hierárquico (HCA) e a análise de componentes principais (PCA).

A HCA é usada para classificar objetos (amostras) pelos valores de um conjunto de variáveis, dentro de um grupo. Com este método, as amostras ou variáveis são agrupadas de acordo com suas similaridades. Os resultados são apresentados na forma de dendrograma, permitindo a visualização das distâncias entre os objetos ou variáveis ${ }^{13}$. Neste diagrama, a escala varia de zero (amostras similares) a 25 (amostras sem similaridades).

A PCA é uma técnica de análise multivariada baseada nas combinações lineares das variáveis originais. O novo conjunto de variáveis, denominadas componentes principais, são ortogonais entre si e, portanto, não correlacionadas. As primeiras componentes prin- 
cipais explicam a maior parte da variância total contida no conjunto de dados e podem ser usadas para representá-lo.

Do ponto de vista matemático a PCA decompõe uma matriz de dados brutos representada por $\mathrm{X}$, em duas matrizes, uma de escores ("scores") T e a outra de pesos ("loadings") $\mathrm{L}^{\mathrm{T}}$, ou seja $\mathrm{X}=\mathrm{T} . \mathrm{L}^{\mathrm{T}}$, onde os escores são as projeções das amostras na direção das componentes principais (CP), enquanto que os pesos são os cossenos dos ângulos formados entre a componente principal e cada variável original ${ }^{14,15}$.

Neste trabalho, as técnicas de análises multivariadas (HCA e PCA) foram aplicadas ao conjunto de dados formados por 13 variáveis medidas em 19 amostras de solos urbanos da cidade de Teresina, visando identificar os locais poluídos por metais pesados e distinguir a contribuição natural da antrópica.

\section{PARTE EXPERIMENTAL}

\section{Área estudada e amostragem dos solos}

A cidade de Teresina (Figura 1) está localizada na região centro-norte do estado do Piauí e meio-norte do nordeste brasileiro, com latitude sul $05^{\circ} 05^{\prime} 12^{\prime \prime}$ e longitude oeste $42^{\circ} 48^{\prime} 42^{\prime \prime}$. Ocupa uma área superficial de $1679,8 \mathrm{~km}^{2}$, com aproximadamente 715.000 habitantes, sendo aproximadamente 676.900 residentes na área urbana e os demais na área rural e uma densidade demográfica de 425,6 hab $/ \mathrm{km}^{2}$. A cidade é banhada por dois rios, Parnaíba e Poti, e foi fundada em 1852 .

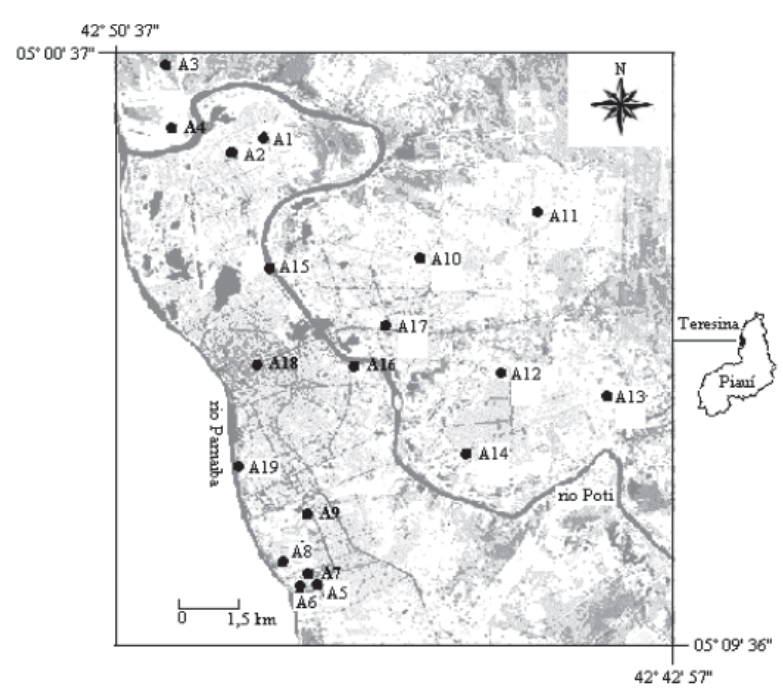

Figura 1. Mapa da área estudada, com a localização dos pontos de amostragem

As amostras de aproximadamente $1 \mathrm{~kg}$ foram obtidas coletando-se 4 a 5 amostras simples (profundidade de 0 a $10 \mathrm{~cm}$ ) para formar uma amostra composta da camada superficial de parques ambientais, avenidas, hortas comunitárias, áreas industriais e áreas residenciais de Teresina. Os locais de amostragem (Figura 1) foram selecionados, levando-se em consideração às áreas que apresentem ou não a perturbação humana. As descrições dos locais amostrados estão indicadas na Tabela 1. Todas as amostras foram coletadas com equipamento de plástico, secadas ao ar por $24 \mathrm{~h} \mathrm{e}$ peneiradas em peneiras de polietileno com malha de $2 \mathrm{~mm}$ de diâmetro. A partir deste material foram realizadas as análises químicas e digestões para quantificação dos metais.
Tabela 1. Localização dos pontos de coletas das amostras de solo na cidade de Teresina

\begin{tabular}{|c|c|}
\hline AMOSTRA & LOCALIZAÇÃO \\
\hline A1 & $\begin{array}{l}\text { Pontos na região central do Parque do Buenos Aires } \\
\text { ou Antigo Horto Florestal, está instalado numa área } \\
\text { de } 38 \text { hectares. Av. Freitas Neto no bairro } \\
\text { Mocambinho. }\end{array}$ \\
\hline A2 & $\begin{array}{l}\text { Pontos próximos ao muro do Parque do Buenos } \\
\text { Aires ou Antigo Horto Florestal. }\end{array}$ \\
\hline A3 & Terreno próximo ao bairro Santa Maria da Codipi. \\
\hline A4 & $\begin{array}{l}\text { Terreno próximo da ponte Mariano Gayoso Castelo } \\
\text { Branco, depois do bairro Poti Velho. }\end{array}$ \\
\hline A5 & $\begin{array}{l}\text { Terreno próximo ao bairro Parque Piauí e distrito } \\
\text { industrial. }\end{array}$ \\
\hline
\end{tabular}

Terreno de uma fábrica de caixa de fibra no distrito industrial.

A7

Terreno próximo ao bairro Saci.

A8 Campo de futebol da antiga fábrica de soro fisiológico (Insopisa).

A9 Horta comunitária em frente à subestação da CHESF e ao lado de uma fábrica de produtos alimentícios (Mapil).

A10 Terreno próximo ao Horto Florestal, situado na rua Visconde da Parnaíba.

A11 Parque Mão Santa, próximo ao campo de futebol e ao mercado, depois do bairro Piçarreira.

A12 Avenida Joaquim Nelson, próxima ao Bairro Itararé. A13 Horta comunitária próxima ao bairro Renascença.

A14 Horta comunitária próxima à cerâmica Fortes, na vila são Lázaro.

A15 Nas margens, ao longo da avenida Marechal Castelo Branco. Avenida situada na margem esquerda do rio Poti.

A16 Parque Municipal Floresta Fóssil situado na margem direita do rio Poti.

A17 Nos canteiros e terrenos ao longo da avenida João XXIII. Avenida de continuação da avenida Frei Serafim, ligando-a com o acesso a zona norte do estado.

A18 Nos canteiros, ao longo da Avenida Frei Serafim. Avenida central da cidade de Teresina.

A19

Nas margens, ao longo da avenida Maranhão. Avenida situada na margem direita do rio Parnaíba.

\section{Soluções e reagentes}

Todos os reagentes usados na digestão foram de grau analítico e a água utilizada na preparação de todas as soluções de trabalho foi obtida através de um sistema de purificação Milli-Q (Millipore, modelo RO15), resistividade 18,2 $\mathrm{M} \Omega \mathrm{cm}^{-1}$. Os ácidos $\mathrm{HCl}$ e $\mathrm{HNO}_{3}$ utilizados na digestão foram de qualidade suprapur-Merck. As vidrarias e os frascos de polietileno permaneceram imersos em solução de ácido nítrico 10\% PA (Merck) durante 24 h. Em seguida, foram enxaguados com água deionizada e colocados para secagem à temperatura ambiente. As soluções padrão dos elementos usadas na calibração do aparelho de espectrometria de absorção atômica foram preparadas, diluindo-se uma solução estoque de $1000 \mathrm{mg} \mathrm{L}^{-1}$ ( $\mathrm{Pb}, \mathrm{Co}, \mathrm{Cr}, \mathrm{Ni}, \mathrm{Al}, \mathrm{Cu}, \mathrm{Cd}, \mathrm{Mg}, \mathrm{Zn}$ ) e $1026 \mathrm{mg} \mathrm{L}^{-1}$ (Fe), fornecido pela Fluka e ampola $1000 \mathrm{mg}(\mathrm{Mn})$ fornecida pela Merck. 


\section{Procedimento de digestão}

Uma massa de $1,5 \mathrm{~g}$ de solo foi transferida para um balão de 50 $\mathrm{mL}$, adicionaram-se $2 \mathrm{~mL}$ de $\mathrm{HNO}_{3}$ e $6 \mathrm{~mL}$ de $\mathrm{HCl}$ e tampou-se com funil. A primeira etapa foi a pré-digestão realizada à temperatura ambiente por $16 \mathrm{~h}$ e, em seguida, a digestão foi realizada por $4 \mathrm{~h}$ á temperatura aproximada de $100{ }^{\circ} \mathrm{C}$. O resíduo foi filtrado e diluído para $25 \mathrm{~mL}$ com água deionizada. As soluções não ficaram claras. As análises foram realizadas em duplicatas. Dois brancos foram realizados seguindo as mesmas condições para lotes de seis amostras digeridas.

\section{Análises químicas dos solos}

Os valores de $\mathrm{pH}$ em água foram obtidos segundo o Manual de Métodos e Análises Químicas de Solos da Embrapa ${ }^{16}$, empregando-se a relação 1:2,5 de solo:água. As demais análises seguiram os procedimentos descritos por Raij e colaboradores ${ }^{17}$. Os valores de $\mathrm{pH}$ em $\mathrm{CaCl}_{2}$ 0,01 mol L-1 foram obtidos potenciometricamente em suspensão, empregando-se a relação 1:2,5 de solo: $\mathrm{CaCl}_{2}$. Os

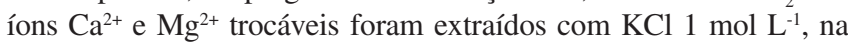
proporção $1: 10$, sendo determinado por titulação com solução padronizada de EDTA $0,005 \mathrm{~mol} \mathrm{~L}^{-1}$. A acidez total $\left(\mathrm{H}^{+}+\mathrm{Al}^{3+}\right)$ foi determinada com acetato de cálcio $1 \mathrm{~mol} \mathrm{~L}^{-1}$, ajustado o $\mathrm{pH}$ para 7,0, na proporção 1:20 de solo:solução, sendo determinada por titulação com solução padronizada de $\mathrm{NaOH} 0,025 \mathrm{~mol} \mathrm{~L}^{-1}$.

\section{Análise instrumental por espectrometria de absorção atômica}

Nas amostras digeridas foram analisados os elementos $\mathrm{Pb}, \mathrm{Cd}$, $\mathrm{Co}, \mathrm{Cr}, \mathrm{Ni}, \mathrm{Fe}, \mathrm{Al}, \mathrm{Cu} \mathrm{Zn}$ e Mn por espectrometria de absorção atômica em chama (Varian, SpectrAA 220 FS). Os parâmetros operacionais do aparelho são mostrados no Tabela 2.

Tabela 2. Condições operacionais do espectrômetro de absorção atômica

\begin{tabular}{lcccl}
\hline Elemento & $\lambda^{\mathrm{a}}(\mathrm{nm})$ & $\mathrm{F}^{\mathrm{b}}(\mathrm{nm})$ & $\mathrm{CL}^{\mathrm{c}}(\mathrm{mA})$ & Tipo de chama \\
\hline $\mathrm{Cu}$ & 324,7 & 0,5 & 4,0 & ar-acetileno \\
$\mathrm{Pb}$ & 217,0 & 1,0 & 5,0 & ar-acetileno \\
$\mathrm{Zn}$ & 213,9 & 1,0 & 5,0 & ar-acetileno \\
$\mathrm{Cr}$ & 357,9 & 0,2 & 7,0 & ar-acetileno \\
$\mathrm{Ni}$ & 232,0 & 0,2 & 4,0 & ar-acetileno \\
$\mathrm{Al}$ & 309,3 & 0,5 & 10,0 & óxido nitroso-acetileno \\
$\mathrm{Mn}$ & 403,1 & 0,2 & 5,0 & ar-acetileno \\
$\mathrm{Fe}$ & 248,3 & 0,2 & 5,0 & ar-acetileno \\
$\mathrm{Co}$ & 240,7 & 0,2 & 7,0 & ar-acetileno \\
$\mathrm{Cd}$ & 228,8 & 0,5 & 4,0 & ar-acetileno \\
\hline
\end{tabular}

${ }^{\mathrm{a}}$ Comprimento de onda; ${ }^{\mathrm{b}}$ abertura da fenda; ${ }^{\mathrm{c}}$ corrente aplicada à lâmpada.

As análises estatísticas foram processadas utilizando o software SPSS (Statistical Package for the Social Sciences) e os gráficos foram elaborados usando o software Origin.

\section{RESULTADOS E DISCUSSÃO}

As características químicas da fertilidade dos solos coletados na área urbana de Teresina são apresentadas na Tabela 3.

Os valores obtidos para $\mathrm{pH}$ em água e $\mathrm{pH}$ em $\mathrm{CaCl}_{2}$ variaram de 4,36 na amostra (A1) a 7,79 na amostra (A10) e 3,73 na amostra (A1) a 7,48 na amostra (A10), respectivamente. Os valores de $\mathrm{pH}$
Tabela 3. Características químicas da fertilidade do solo

\begin{tabular}{llcrc}
\hline Variáveis & Mínimo & Máximo & Média & $\mathrm{CV}^{\mathrm{b}}(\%)$ \\
\hline $\mathrm{pH}\left(\mathrm{H}_{2} \mathrm{O}\right)$ & $4,36(\mathrm{~A} 1)$ & $7,79(\mathrm{~A} 10)$ & 6,35 & 14,67 \\
$\mathrm{pH}\left(\mathrm{CaCl}_{2}\right)$ & $3,73(\mathrm{~A} 1)$ & $7,48(\mathrm{~A} 10)$ & 5,99 & 16,98 \\
$\mathrm{Ca}^{2+\mathrm{a}}$ & $2,00(\mathrm{~A} 1)$ & $83,00(\mathrm{~A} 6)$ & 44,95 & 46,22 \\
$\mathrm{Mg}^{2+\mathrm{a}}$ & $1,00(\mathrm{~A} 8)$ & $32,00(\mathrm{~A} 17)$ & 7,00 & 96,07 \\
$\mathrm{H}^{+}+\mathrm{Al}^{3+\text { a }}$ & $2,00(\mathrm{~A} 10)$ & $40,00(\mathrm{~A} 18)$ & 18,68 & 60,84 \\
\hline
\end{tabular}

${ }^{\mathrm{a}} \mathrm{mmol} \mathrm{dm}^{-3} ;{ }^{\mathrm{b}}$ coeficiente de variação.

são úteis para classificar a acidez ativa, desde solos muito ácidos $(\mathrm{pH}<4,50)$ a solos fracamente alcalinos $\left(\mathrm{pH}\right.$ entre 7,10- 7,80) ${ }^{18}$. As amostras coletadas no parque do Buenos Aires (A1 e A2) são classificadas como muito ácidas. A amostra do terreno na Av. Visconde da Parnaíba (A10), próximo ao Horto Florestal, é classificada como fracamente alcalina.

A acidez total $\left(\mathrm{H}^{+}+\mathrm{Al}^{3+}\right)$ dos solos analisados apresentou valores variando entre 2,00 $\mathrm{mmol}_{\mathrm{c}} \mathrm{dm}^{-3}$ na amostra (A10) e 40,00 $\mathrm{mmol}$ c

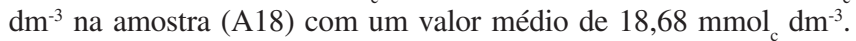
Nenhuma das amostras analisadas apresentou a acidez total alta (> 50,00 $\mathrm{mmol}_{\mathrm{c}} \mathrm{dm}^{-3}$ ), sendo que todas tiveram a classificação da acidez entre muito baixo $\left(\leq 10 \mathrm{mmol}_{\mathrm{c}} \mathrm{dm}^{-3}\right)$ e média (entre 25,10 a $50,00 \mathrm{mmol} \mathrm{dm}^{-3}$ ), de acordo com os critérios de fertilidade dos solos adotados no Estado de Minas Gerais ${ }^{18}$.

Nos solos que apresentam elevada acidez, ocorre dissolução de alumínio, que passa a ser um componente da acidez total. O alumínio é a principal causa da acidez excessiva dos solos, podendo tornar-se um elemento fitotóxico. Em condições de acidez elevada também pode ocorrer a solubilização de outros metais, como os metais pesados, disponibilizando-os para absorção pelas plantas.

Este parâmetro também é importante para definir a necessidade ou não de correção do solo através de calagem, sendo que a necessidade não está relacionada somente ao $\mathrm{pH}$ do solo, mas também à sua CTC, onde as quantidades totais de argila e de matéria orgânica em um solo determinam o poder tampão deste solo, ou seja, a resistência à mudança de $\mathrm{pH}$. A capacidade tampão de um solo aumenta com a quantidade de argila e de matéria orgânica presentes, estes solos necessitam de uma quantidade maior de calcário para aumentar o seu $\mathrm{pH}$, enquanto que solos com baixa capacidade tampão (solos arenosos) necessitam de uma quantidade menor de calcário. As amostras com maior acidez total foram aquelas coletadas na Av. Frei Serafim (A18) e no parque do Buenos Aires (A1).

$\mathrm{O}$ cálcio e o magnésio trocáveis apresentaram valores variando de $2,00 \mathrm{mmol} \mathrm{dm}^{-3}$ na amostra (A1) a $83,00 \mathrm{mmol} \mathrm{dm}^{-3}$ na amostra (A6) e $1 \mathrm{mmol}_{\mathrm{c}} \mathrm{dm}^{-3}$ na amostra (A8) a $32 \mathrm{mmol} \mathrm{dm}^{-3}$ na amostra (A17), respectivamente. O solo que apresentou menor teor de cálcio trocável foi o situado no parque do Buenos Aires (A1) e o maior foi o solo coletado em um terreno no parque industrial, onde funciona uma fábrica de caixas de fibra (A6). A quantidade de cálcio é maior nos solos argilosos que nos arenosos, podendo o cálcio trocável estar adsorvido às superfícies das argilas e da matéria orgânica. $O$ cálcio também está presente na solução do solo e nas estruturas de vários minerais, como dolomita, calcita, apatita e feldspatos cálcicos ${ }^{19}$. O magnésio do solo além daquele adicionado por fertilizantes ou corretivos, também pode ser oriundo da intemperização das rochas. Os solos, geralmente contêm menos magnésio que cálcio, porque o magnésio não é adsorvido tão fortemente pela argila e pela matéria orgânica, sendo sujeito à lixiviação ${ }^{19}$.

A adição de cálcio em um solo deficiente em magnésio pode causar desequilíbrio nutricional, reduzindo o crescimento das plantas. 
Tabela 4. Limite de detecção e parâmetros estatísticos relativos aos metais analisados por espectrometria de absorção atômica

\begin{tabular}{|c|c|c|c|c|c|}
\hline Elemento & LD (mg/L) & Mínimo (mg/kg) & Máximo (mg/kg) & Média (mg/kg) & $\mathrm{CV}(\%)$ \\
\hline $\mathrm{Cu}$ & 0,014 & $1,25(\mathrm{~A} 1)$ & 17,13 (A13) & 6,11 & 68,74 \\
\hline $\mathrm{Pb}$ & 0,027 & $1,50(\mathrm{~A} 1)$ & $36,36(\mathrm{~A} 15)$ & 8,56 & 105,96 \\
\hline $\mathrm{Zn}$ & 0,001 & $4,77(\mathrm{~A} 1)$ & 93,31 (A18) & 32,12 & 83,16 \\
\hline $\mathrm{Cr}$ & 0,019 & 3,00 (A14) & $11,10(\mathrm{~A} 10)$ & 7,17 & 36,96 \\
\hline $\mathrm{Ni}$ & 0,016 & $<\mathrm{LD}$ & 3,32 (A16) & 0,87 & 114,14 \\
\hline $\mathrm{Al}$ & 0,300 & 2957,09 (A17) & 8161,30 (A9) & 5469,14 & 27,18 \\
\hline $\mathrm{Mn}$ & 0,057 & 21,13 (A1) & 161,27 (A16) & 65,63 & 63,48 \\
\hline $\mathrm{Fe}$ & 0,316 & $4484,40(\mathrm{~A} 8)$ & $13550,21(\mathrm{~A} 15)$ & 10823,44 & 21,67 \\
\hline Co & 0,037 & $<\mathrm{LD}$ & & & \\
\hline $\mathrm{Cd}$ & 0,011 & $<\mathrm{LD}$ & & & \\
\hline
\end{tabular}

$\mathrm{LD}=$ Limite de detecção experimental; $\mathrm{CV}=$ Coeficiente de variação

O limite de detecção calculado e os principais parâmetros estatísticos obtidos para os metais analisados por espectrometria de absorção atômica são apresentados na Tabela 4.

Os elementos Co e Cd foram analisados e não constam na Tabela 4, pois suas concentrações encontram-se abaixo do limite de detecção calculado para o método.

Para o níquel sete amostras também apresentaram concentrações menores que o limite de detecção calculado. Os maiores coeficientes de variação (Tabela 4) foram observados para os elementos $\mathrm{Ni}$ e $\mathrm{Pb}$, enquanto que os elementos $\mathrm{Fe}$ e $\mathrm{Al}$ apresentaram as menores variabilidades nas concentrações. A variabilidade pode ser útil para identificar padrões na distribuição da concentração destes elementos. Os maiores teores de $\mathrm{Pb}(>17 \mathrm{mg} / \mathrm{kg}$ ) foram observados nas amostras coletadas nas proximidades de avenidas importantes de Teresina (A15, A18 e A19) e os menores teores (< $2,5 \mathrm{mg} / \mathrm{kg}$ ) foram verificados em amostras coletadas em parques ambientais e terrenos da cidade (A1, A7, A2 e A5). Os maiores teores de $\mathrm{Ni}(>2,5 \mathrm{mg} / \mathrm{kg}$ ) estão situados nas proximidades do rio Poti (A4 e A16) e no terreno de uma fábrica de caixas de fibra (A6), representando, possivelmente, a contribuição natural e antrópica, respectivamente.

\section{Análise de componentes principais}

A análise de componente principal foi realizada sobre a matriz de dados composta de 13 variáveis e 19 amostras de solos de Teresina, onde os dados foram previamente autoescalados (média zero e variância igual a 1), um vez que existe uma grande variação das respostas das diversas variáveis, ou seja, diferem em ordem de grandeza.

Às amostras com teor de analito abaixo do limite de detecção do método, foi atribuída concentração igual a zero. Dentre as 13 componentes principais geradas, as quatro primeiras descreveram aproximadamente $79 \%$ da variância total dos dados. Estas quatro componentes foram giradas através da rotação varimax ${ }^{20,21}$, para facilitar a interpretação da contribuição das variáveis (pesos) em cada componente principal.

Na Tabela 5, são apresentados somente os pesos das componentes principais rotacionadas maiores que 0,500 e menores que -0,500 para facilitar a identificação das variáveis com maior importância na combinação linear de cada componente. $\mathrm{O}$ magnésio trocável não foi incluído na Tabela 5 porque os pesos mais significativos que apresentou foram 0,404 na componente 3 e $-0,453$ na componente 4.

A primeira componente principal explica $25,42 \%$ da variância total dos dados. Os maiores pesos positivos nesta componente são os das variáveis $\mathrm{pH}$ em água, $\mathrm{pH}$ em $\mathrm{CaCl}_{2} \mathrm{e} \mathrm{Ca}^{2+} \mathrm{e}$ o maior peso
Tabela 5. Pesos das variáveis nas componentes principais (CP) após rotação varimax

\begin{tabular}{lrrrr}
\hline & \multicolumn{4}{c}{ CP rotacionadas } \\
\cline { 2 - 5 } Variáveis & $\mathrm{CP} 1$ & $\mathrm{CP} 2$ & $\mathrm{CP} 3$ & $\mathrm{CP} 4$ \\
\hline $\mathrm{pH}\left(\mathrm{H}_{2} \mathrm{O}\right)$ & 0,938 & & \\
$\mathrm{pH}\left(\mathrm{CaCl}_{2}\right)$ & 0,939 & & & \\
$\mathrm{Ca}^{2+}$ & 0,688 & & & \\
$\mathrm{H}^{+}+\mathrm{Al}^{3+}$ & $-0,879$ & & & \\
$\mathrm{Cu}$ & & 0,746 & & \\
$\mathrm{~Pb}$ & & 0,824 & & \\
$\mathrm{Zn}$ & 0,883 & & \\
$\mathrm{Cr}$ & & & 0,809 & \\
$\mathrm{Ni}$ & & & 0,581 & \\
$\mathrm{Al}$ & & & 0,932 \\
$\mathrm{Mn}$ & & & 0,846 & \\
$\mathrm{Fe}$ & & & & \\
\hline
\end{tabular}

negativo é observado apenas na variável acidez total $\left(\mathrm{H}^{+}+\mathrm{Al}^{3+}\right)$, sugerindo que essa componente principal representa as propriedades químicas que caracterizam a fertilidade de um solo.

A segunda componente principal explica $21,63 \%$ da variância total dos dados e os maiores pesos são observados nas variáveis ( $\mathrm{Cu}, \mathrm{Pb}$ e $\mathrm{Zn}$ ). Dependendo de suas concentrações, estes elementos podem estar associados à contaminação antrópica dos solos urbanos ${ }^{9,12,22,23}$. Portanto, a componente principal 2 pode ser interpretada como responsável pela contribuição antrópica ao solo.

A terceira componente principal explica $17,14 \%$ da variância total dos dados e possui pesos positivos nas variáveis $(\mathrm{Cr}, \mathrm{Fe}$ e $\mathrm{Al})$, sendo que os óxidos de ferro e alumínio estão presentes naturalmente em todos os solos. Portanto, esta componente principal está associada à contribuição natural de metais ao solo.

A quarta componente principal explica $14,87 \%$ da variância total dos dados apresenta pesos positivos nas variáveis (Mn e Ni). Os óxidos de manganês, quando encontrados em formas não cristalinas podem adsorver metais ${ }^{24}$, não sendo observado esse tipo de associação, pois apenas o níquel apresentou correlação com o manganês significativa estatisticamente $(0,754)$.

A Figura 2 mostra o gráfico das duas primeiras componentes principais. O gráfico dos escores da componente principal 1 versus os da componente principal 2 explica $47,05 \%$ da variância total dos dados. A amostra do parque do Buenos Aires (A1) apresentou os maiores valores de acidez total e os menores valores de $\mathrm{pH}\left(\mathrm{H}_{2} \mathrm{O}\right.$ e $\mathrm{CaCl}_{2}$ ) e de $\mathrm{Ca}^{2+}$, localizando-se na parte negativa da componente principal 1 e foi classificada como muito ácida. A amostra de um 


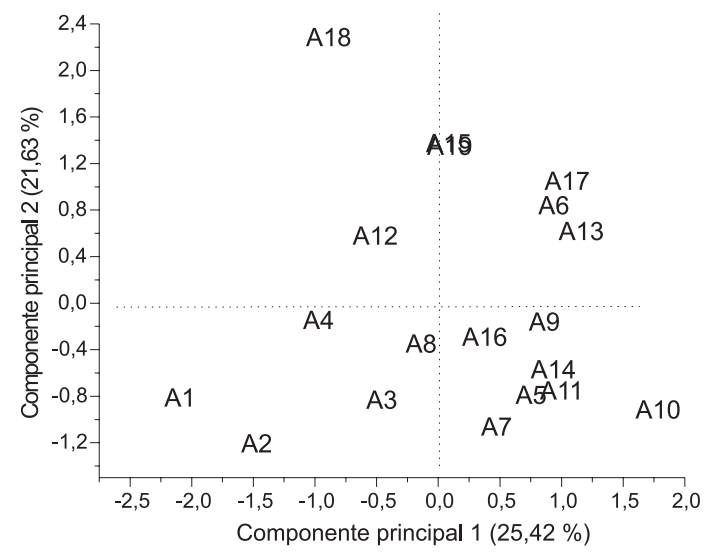

Figura 2. Gráfico dos escores da componente principal 1 versus componente principal 2

terreno na R. Visconde da Parnaíba próximo ao Horto Florestal (A10) destacou-se na parte positiva da componente principal 1 , por apresentar menor valor para acidez total e o maior valor de $\mathrm{pH}$. A amostra A10 é classificada como fracamente alcalina. Segundo o eixo da componente principal 1 é possível ordenar todas as amostras por sua fertilidade.

A amostra coletada nos canteiros ao longo da Av. Frei Serafim (A18) destaca-se das demais amostras na parte positiva da componente principal 2, devido à maior contribuição antrópica. Esta amostra apresentou a maior concentração de $\mathrm{Zn}$. As amostras coletadas nas proximidades das Av. Mal. Castelo Branco (A15) e Av. Maranhão (A19) destacaram-se na componente 2 por apresentarem altas concentrações dos elementos $\mathrm{Zn}$ e $\mathrm{Pb}$. Na Figura 2 estas amostras (A15 e A19) encontram-se praticamente sobrepostas. A Av. Mal. Castelo Branco (A15), que acompanha o percurso do rio Poti apresentou concentrações de $\mathrm{Zn}$ e Pb iguais a 40,80 e 36,36 $\mathrm{mg} / \mathrm{kg}$, respectivamente e a Av. Maranhão (A19), que acompanha o percurso do rio Parnaíba, concentrações de $\mathrm{Zn}$ e Pb iguais a 53,72 e $17,34 \mathrm{mg} / \mathrm{kg}$, respectivamente.

A amostra coletada em um terreno de uma fábrica de caixa de fibra (A6) apresentou concentrações de $\mathrm{Cu}$ e $\mathrm{Zn}$ maiores que as determinadas nas amostras das Avs. Joaquim Nelson (A12) e Mal. Castelo Branco (A15), valor da concentração de Pb próxima da determinada na amostra (A12) e menor que metade do valor determinado na amostra (A15), portanto a componente principal 2 pode ser entendida como classificadora da contribuição antrópica do solo ficando, na parte negativa do eixo, os solos isentos de contaminação e, na parte positiva, os solos possivelmente contaminados.

As maiores concentrações de $\mathrm{Pb}$ nos solos urbanos foram determinadas em amostras coletadas próximo a avenidas antigas, que possuem grande tráfego de veículos. Segundo a literatura ${ }^{25,26}$, isto pode ser atribuído a queima de gasolina, contendo uma substância anti-detonante, denominada chumbo tetraetila. No Brasil essa substância foi substituída pelo álcool, deixando de ser um dos principais responsáveis pela contaminação veicular. $\mathrm{O} \mathrm{Pb}$ é um dos metais pesados que apresenta menor mobilidade ${ }^{3}$ e as quantidades determinadas nestes solos podem estar relacionadas às deposições ocorridas antes da substituição do $\mathrm{Pb}$ da gasolina. Isto é corroborado pelo menor teor de $\mathrm{Pb}$ determinado em amostras próximas de avenidas mais recentes como, por ex., a Av. Joaquim Nelson (A12) construída após a substituição desta substância na gasolina. A amostra A12 apresentou concentração de $\mathrm{Pb}$ maior que a concentração determinada nos solos coletados nos parques urbanos (A1 e A2), terrenos (A3, A4, A7, A10 e A11) e hortas comunitárias (A9 e A14), sugerindo que ainda pode existir alguma fonte de poluição deste elemento.

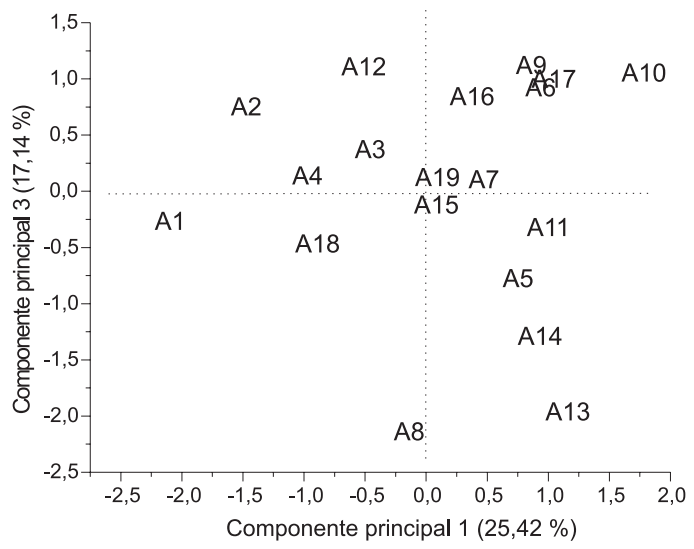

Figura 3. Gráfico dos escores da componente principal 1 versus componente principal 3

A Figura 3 mostra o gráfico dos escores da primeira com a terceira componente principal, que explica $42,56 \%$ da variância total dos dados. A amostra coletada ao longo da Av. Joaquim Nelson (A12) apresentou elevada concentração de Fe; a amostra coletada em um terreno na R. Visconde da Parnaíba (A10), maior concentração de Cr e a amostra de uma horta comunitária (A9) a maior concentração de Al. As amostras coletadas na Av. Joaquim Nelson (A12) e na horta próxima a fábrica de produtos alimentícios Mapil (A9) destacaram-se na parte positiva da componente principal 3, apresentando valores das concentrações de $\mathrm{Cr}, \mathrm{Fe}$ e Al próximos e as amostras coletadas em um campo de futebol (A8) e na horta próxima ao bairro Renascença (A13) destacaram-se na parte negativa desta componente por apresentarem baixas concentrações de $\mathrm{Fe}, \mathrm{Cr}$ e $\mathrm{Al}$, sendo que as concentrações de ferro são as menores de todas as amostras analisadas. $\mathrm{O}$ eixo da componente principal 3 reflete apenas a intensidade da contribuição natural à composição mineral do solo, portanto na parte negativa deste eixo ficam as amostras pobres nestes metais ( $\mathrm{Fe}, \mathrm{Cr}$ e $\mathrm{Al}$ ).

A Figura 4 mostra o gráfico dos escores da primeira com a quarta componente principal, o qual explica $40,25 \%$ da variância total dos dados. A amostra coletada no Parque Municipal Floresta Fóssil (A16) apresentou as maiores concentrações de $\mathrm{Mn}$ e Ni, destacandose na parte positiva da componente principal 4. As amostras coletadas próximo à ponte Mariano Gayoso Castelo Branco (A4) e no terreno

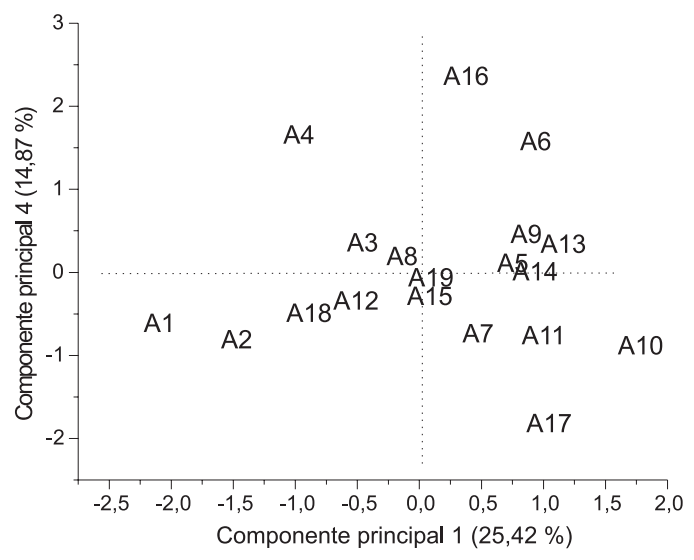

Figura 4. Gráfico dos escores da componente principal 1 versus componente principal 4 
da fábrica de caixas de fibras (A6) apresentaram valores próximos para as concentrações de Ni, sendo observada uma pequena diferença entre os valores das concentrações de manganês. Embora não haja dúvida quanto a contribuição antrópica na amostra A6, nada se pode dizer das amostras A16 e A4.

\section{Análise de agrupamento hierárquico}

A análise de agrupamento hierárquico foi aplicada à matriz dos escores das quatro componentes principais rotacionadas, gerando um dendrograma que fornece uma visão bidimensional de $79 \%$ da informação estatística do sistema. Para sua construção não houve necessidade de pré-tratamento dos dados. Utilizou-se como medida de similaridade o quadrado da distância euclidiana e, para delimitação dos grupos foi utilizado o método da distância média entre grupos. No dendrograma mostrado na Figura 5 destacam-se cinco grupos. Existem cinco amostras no grupo 1, quatro no grupo 2, duas nos grupos 3 e 5, e seis no grupo 4 .

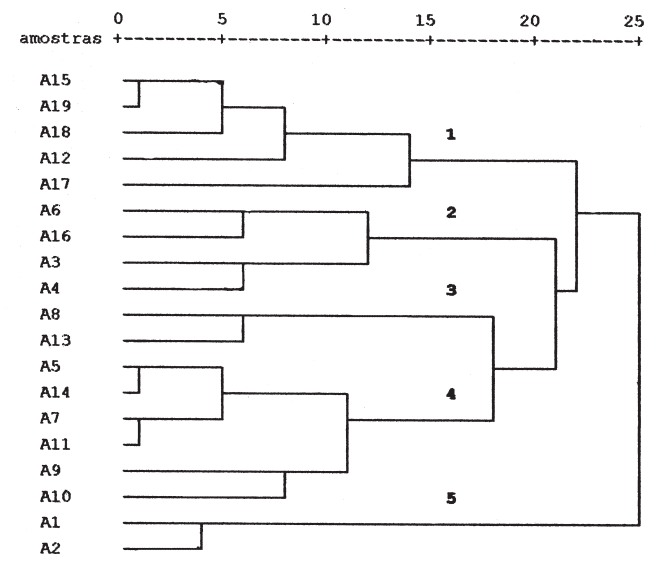

Figura 5. Dendrograma obtido na análise de agrupamento hierárquico à matriz dos escores das componentes

No grupo 1, tem-se as amostras A15, A19, A18, A12 e A17, que foram coletadas nas proximidades de avenidas. As amostras A15 e A19 apresentaram as maiores similaridades deste grupo, pois são provenientes de avenidas mais antigas, que possuem grande tráfego de veículos, ou seja, contribuições antrópicas similares, apresentando altas concentrações para os metais $\mathrm{Cu}, \mathrm{Zn}$, e $\mathrm{Pb}$, podendo ser resultante de atividades antrópicas. Resultado semelhante foi observado na PCA, podendo ser verificado no gráfico de escores da Figura 2.

O grupo 2 é formado pelas amostras A6, A16, A3 e A4 e caracteriza-se por elevados teores de $\mathrm{Mn}$ e Ni. Em geral, estes dois metais não apresentam origem antrópica e sim, na geoquímica das rochas ${ }^{27}$. As amostras A16 e A4 foram coletadas próximo das margens do rio Poti e têm maior relação com a contribuição natural. A semelhança entre as amostras A3 e A4 está relacionada com a proximidade dos pontos de coleta, apresentando pouca perturbação humana. A amostra A6 apresenta maior relação com a contribuição antrópica, por ser proveniente de um terreno que apresenta grandes quantidades de materiais depositados na superfície, em decorrência da produção das caixas de fibras.

O grupo 3 é formado apenas pelas amostras A8 e A13; a similaridade destas amostras está relacionada com as concentrações de $\mathrm{Cr}, \mathrm{Al}$ e $\mathrm{Fe}$, pois as duas amostras foram coletadas em locais distantes um do outro, sendo a similaridade atribuída aos baixos valores de concentração dos elementos $\mathrm{Cr}, \mathrm{Al}$ e $\mathrm{Fe}$. Os teores de ferro são os menores quando comparados com todas as amostras analisadas.

O grupo 4 é formado por solos de terrenos urbanos (A5, A7, A11 e A10) e de hortas comunitárias (A9 e A14); as maiores similaridades foram observadas para as amostras A5 e A14 e para A7 e A11, onde as proximidades nos valores das concentrações de $\mathrm{Cr}$, Fe e Al foram responsáveis pelo grau de similaridade observada no dendrograma (Figura 5). Este grupo apresenta amostras com acidez total muito baixa $\leq 10 \mathrm{mmol}_{\mathrm{c}} \mathrm{dm}^{-3}$, podendo ser representado por amostras que possivelmente apresentam modificações nas concentrações metálicas de ambas as fontes, natural e antrópica, visto que alguns terrenos não apresentam indícios de contaminação antrópica (aumento na concentração de metais pesados), enquanto que as hortas comunitárias estão mais sujeitas a esse tipo contaminação devido à adição de fertilizantes, que contêm metais pesados em sua composição ${ }^{5}$ e podem acumular no solo, aumentado sua disponibilidade para plantas.

Finalmente, o grupo 5 é formado pelas amostras coletadas no Parque Ambiental do Buenos Aires (A1 e A2). Este grupo é caracterizado por solos que apresentaram baixa fertilidade e baixos teores de metais pesados, indicando a não existência de atividades antrópicas no local, demonstrando uma forte associação com a origem natural.

Os resultados obtidos com a HCA complementam aqueles obtidos por PCA, fornecendo uma visão de conjunto de todas as amostras e como as mesmas se assemelham. Por outro lado, a PCA permitiu melhor interpretação dos grupos naturalmente formados na HCA, pois distinguiu melhor a contribuição de grupos de elementos metálicos, facilitando identificar a contribuição antrópica ou natural em cada caso.

\section{CONCLUSÃO}

As áreas urbanas têm sido bastante afetadas pela atividade humana, verificando-se um aumento na quantidade de metais acumulados na superfície dos solos, como $\mathrm{Cu}, \mathrm{Pb}$ e $\mathrm{Zn}$. O enriquecimento da camada superficial de alguns solos foi atribuído à poluição de fontes antrópicas.

Como a cidade de Teresina não apresenta grandes indústrias, as áreas que indicaram um aumento na concentração de metais pesados, como $\mathrm{Cu}, \mathrm{Pb}$ e $\mathrm{Zn}$, foram os solos coletados nas margens de avenidas com grande tráfego de veículos. A emissão automobilística ocorrida no passado foi provavelmente responsável pelo elevado conteúdo de $\mathrm{Pb}$ nos solos urbanos das avenidas mais antigas de Teresina.

A integração das técnicas PCA e HCA permitiu classificar todas as amostras de solo estudados em Teresina, mostrando que esse tipo de análise de dados permite que se obtenham informações rápidas e eficientes sobre a similaridade entre as amostras, através da visualização gráfica.

\section{REFERÊNCIAS}

1. Sastre, J.; Sahuquillo, A.; Vidal, M.; Rauret, G.; Anal. Chim. Acta 2002, $462,59$.

2. Bradl, H. B.; J. Colloid Interface Sci. 2004, 277, 1.

3. Kabata-Pendias, A.; Pendias, H.; Trace elements in soils and plants, Boca Raton: CRC Press, 1984.

4. Korchak, R. F.; Fanning, D. S.; Soil Sci. 1985, 140, 23.

5. Gonçalves-Jr., A. C.;Luchese, E. B.; Lenzi, E.; Quim. Nova 2000, 23, 173.

6. Tüzen, M.; Microchem. J. 2003, 74, 289.

7. Raij, B. van.; Fertilidade do solo e adubação, Potafos: Piracicaba, 1991.

8. Morselli, L.; Brusori, B.; Passarini, F.; Bernardi, E.; Francaviglia, R.; Gataleta, L.; Marchionni, M.; Aromolo, R.; Benedetti, A.; Olivieri, P.; Environment International 2004, 30, 173. 
9. Gallego, J. L. R.; Ordóñez, A.; Loredo, J.; Environment International 2002, $27,589$.

10. Nriagu, J. O.; Pacyna, J.; Nature 1988, 333, 134.

11. Guvenç, N.; Alagha, O.; Tuncel, G.; Environment International 2003, 29, 631.

12. Li, X.; Lee, S.; Wong, S.; Shi, W.; Thornton, I.; Environ. Pollut. 2004, 129, 113.

13. Moita Neto, J. M.; Moita, G. C.; Quim. Nova 1998, 21, 467.

14. Sena, M. M.; Poppi, R. J.; Frighetto, R. T. S.; Valarini, P. J.; Quim. Nova 2000, 23, 547.

15. Ferreira, M. M. C.; Montanari, C. A.; Gaudio, A. C.; Quim. Nova 2002, $25,439$.

16. Embrapa; Manual de Métodos e Análises Químicas de Solos, Plantas e Fertilizantes, Brasília, 1999.

17. Raij, B. van; Andrada, J. C.; Cantarella, H.; Quaggio, J. A.; Análise química para avaliação da fertilidade de solos tropicais, Campinas, 2001.
18. Comissão de Fertilidade do solo do Estado de Minas Gerais; Recomendações para o uso de corretivos e fertilizantes em Minas Gerais, Viçosa, 1999.

19. Brady, N. C.; Natureza e propriedades dos solos, $9^{\mathrm{a}}$ ed., Livraria Freitas Bastos: Rio de Janeiro, 1979.

20. Sena, M.M.; Frighetto, R.T.S.; Valarini, P.J.; Tokeshi, H.; Poppi, R.J.; Soil Till. Res. 2002, 67, 171.

21. Facchinelli, A.; Sacchi, E.; Mallen, L.; Environ. Pollut. 2001, 114, 313.

22. Li, X.; Liu, P. S.; Appl. Geochem. 2001, 16, 1361.

23. Manta, D. S.; Angelone, M.; Bellanca, A.; Neri, R.; Sprovieri, M.; Sci. Total Environ. 2002, 300, 229.

24. Meurer, E. J.; Fundamentos de química do solo, Porto Alegre, 2000.

25. Lu, Y.; Gong, Z.; Zhang, G.; Burghardt, W.; Geoderma 2003, 115, 101

26. Imperato, M.; Adamo, P.; Naimo, D.;Arienzo, M.; Stanzione, D.; Violante, P; Environ. Pollut. 2003, 124, 247

27. Reis, T. C.; Tese de Doutorado, Universidade de São Paulo, Brasil, 2002. 